\title{
Zur Theorie der linearen Integralgleichungen.
}

Von

T. Carleman in Upsala.

Wenn der Kern einer Fredholmschen Integralgleichung

$$
p(x)-\lambda \int_{a}^{b} K(x, y) \psi(y) d y=\ldots
$$

auf der Diagonale $y=x$ streckenweise unendlich wird, so verlieren die in den Koeffizienten der Reihen $D(\lambda)$ und $D\left(\begin{array}{l}x \\ y\end{array} \lambda\right)$ auftretenden Integrale ihren Sinn. In seiner grundlegenden Arbeit hat Frodholm gezeigt, daß für eine sehr umfassende Klasse $(L)$ derartiger Integralgleichungen (nämlich solcher, für welche ein iterierter $\operatorname{Kern} K^{(v)}(x, y)$ existiert, der eine beschränkte Funktion von $x$ und $y$ ist) dieselben Gesetze gelten wie für Integralgleichungen mit stetigen Kernen ${ }^{1}$ ). Hilbert betrachtete (Gött. Nachrichten 1904) statt der Reihen $D(\lambda)$ und $D\left(\begin{array}{l}\infty \\ y\end{array} \mid \lambda\right)$ die folgenden

$$
\begin{aligned}
& \text { () } K\left(s_{1}, s_{2}\right) \ldots K\left(s_{1} s_{n}\right)
\end{aligned}
$$

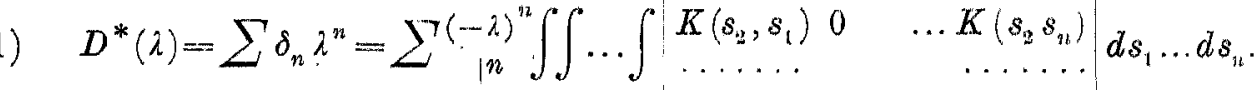

$$
\begin{aligned}
& K\left(s_{i k}, s_{1}\right) \quad \ldots \quad()
\end{aligned}
$$

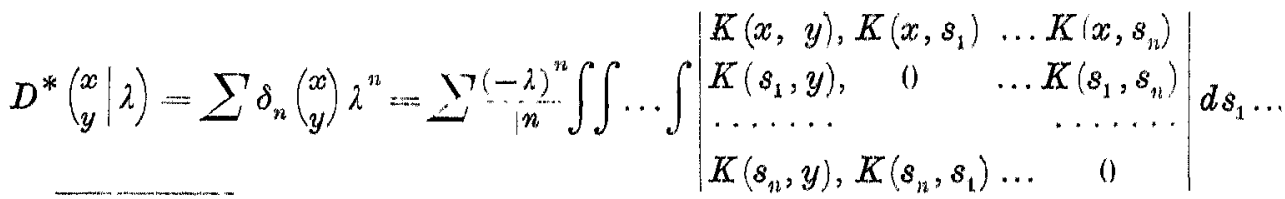

1) Hiermit ist aber die Gesamtheit derjenigen Kerne, für wolche diese Gesetzo gelten, nicht erschöpft, wie das Beispiel

$$
K(x, y)=\sum_{n=2}^{\infty} \frac{\sin n x \cdot \sin n y}{\log ^{2} n} \quad\left(0<\frac{x}{y} \leq \pi\right)
$$

lehrt. Man sieht sofort, daß $K(x, y)$ der Klasse $L$ nicht angehört, weil ja der Konvergenzexponent der zu einem solohen Kerne gehörigen Eigenwerte endlich sein muß. 
Er hat durch direkte Abschätzung der Koeffizienten bewiesen, daß diese Reihen beständig konvergieren, wenn $K(x, y)$ auf der Diagonale $x-y$ von niederer als der der $\frac{1}{3}$ ten Ordnung unendlich wird, $\mathrm{d}$. $\mathrm{h}$. wenn es einen gewissen unterhalb $\frac{1}{\ddot{z}}$ liegenden Exponenten ir gibt, so daß das Produkt

$$
|x-y|^{*} K(x, y)
$$

endlich bleibt. Für $\delta_{n}$ z. B. ergab sich die Ungleichung

$$
\left|\delta_{1}\right|<\frac{c^{n}}{n\left(\frac{1}{2}-x\right)}
$$

Im folgenden wird gezeigt, daß diese Reihen auch dann beständig konvergieren, wenn man nur voraussetzt, daß

$$
\iint|K(x, y)|^{2} d x d y
$$

(im Sinne von Lesbesgue) konvergiert ${ }^{2}$ ). Ferner ergibt sich, dab die Ungleichung (3) durch die schärfere

$$
\left|\partial_{n}\right| \cdots \frac{c^{n}}{n^{\frac{n}{2}}}
$$

ersetzt werden kann. Die Ordnung der ganzen Funktion $D^{*}(\lambda)$ ist somit 52 .

Mit Hilfe dieser Funktionen wird ferner gezeigt, daß die Fredholmschen Sätze auch ferner für solche Integralgleichungen

$$
p(s)-\lambda \int_{a}^{b} K(s, t) q(t) d t=f(s)
$$

gelten, wo $|K(s, t)|^{2}$ und $\left|f^{\prime}(s)\right|^{2}$ summierbare Funktionen sind $\left.{ }^{*}\right)$. Schließlich wird eine Formel betreffend den Fredholmschen Nenner einer Summe von zwei Kernen bewiesen. Aus dieser Formel ergibt sich die Folgerung, dal3 das Geschlecht (Höhe) von $D^{*}(\lambda)$ höchstens gleich Eins sein kann.

\section{$\S 1$.}

In diesem Paragraphen suchen wir obere Grenzen der absoluten Beträge von $D^{*}(\lambda)$ und $D^{*}\left(\begin{array}{l}x \\ y\end{array} \mid \lambda\right)$ unter der Annahme, daß $K(x, y)$ stetig ist and der Nebenbedingung

9) Vgl. hierzu H. von Kooh, Sur la convergence de determinants infinis, Rend. del circ. mat. di Palcrmo, 28 (1209), \$. 255-266 insb. S. 264.

3) Es ist klar, dah dieses Resultat duroh vicle andere Methoden abgeleitet werden kann. Das hier angewandto Verfahren beweist die Existenz einer quadratisch integrierbaron Resolventen, die als Quotient der Funktionen (2) und (1) dargestellt werden bann. Vgl. auoh Lesbesgue, Bull. de la Soo. mathematique de France 36 (1908), S. 3-19. 


$$
\int_{a}^{b} \int_{a}^{b}|K(x, y)|^{2} d x d y \leqq \Omega
$$

genügt. Ohne die Allgemeinheit zu beeinträchtigen, können wir hier annehmen, daß $K(x, y)$ in bezug auf $x$ oder $y$ eine stetige Ableitung besitzt oder sogar ein Polynom ist, weil es immer eine. Polynomfolge $P_{1}(x, y)$, $P_{2}(x, y), \ldots, P_{n}\left(x, y^{\prime}\right) \ldots$ gibt, so daß gleichmäßig

$$
\lim _{n=\infty} P_{n}(x, y)=K(x, y)
$$

und weil ferner

$$
\left.\lim _{n=\infty} D_{P_{n}}^{*}(\lambda)=D_{R}^{*}(\lambda)^{4}\right) \text {. }
$$

Es sei nun $K_{1}(x, y)$ der durch die Festsetzungen

$$
\begin{aligned}
& K_{1}(x, y)=K(x, y) \\
& K_{1}(x, x) \cdots 0
\end{aligned}
$$

definierte Kern. Dann gilt

Weil ferner

$$
D_{K}^{*}(\lambda):=D_{K_{x}}(\lambda)
$$

$$
K_{1}^{(m)}(x, y)=K^{(m)}(x, y)
$$

so folgt aus der Formel

$$
\begin{gathered}
\log D_{K}^{*}(\lambda)=\log D_{K_{1}}(\lambda)=-\sum_{\nu=2}^{\infty} \frac{\lambda^{\nu}}{\nu} \int_{a}^{b} K_{L}^{(\nu)}(s, s) d s \\
=-\sum_{\nu=2}^{\infty} \frac{\lambda^{\nu}}{\nu} \int_{a}^{b} K^{(\nu)}(s, s) d s=\lambda \int_{a}^{b} K(s, s) d s+\log D_{K}(\lambda) \\
D_{K}^{*}(\lambda)=e^{\lambda \int_{\alpha}^{b} K(s, s) d s} D_{K}(\lambda) .
\end{gathered}
$$

Wenn $K(x, y)$ eine stetige Ableitung in bezug auf $x$ oder $y$ besitzt, so ist $D_{K}(\lambda)$ vom Geschlecht Null und folglich gilt

$$
D_{K}(\lambda)=\prod_{\nu=1}^{\infty}\left(1-\frac{\lambda}{\lambda_{\nu}}\right) ; \quad \int_{a}^{b} K(s, s) d s \sum_{\nu=1}^{\infty} \frac{1}{\lambda_{*}},
$$

wo $\lambda_{1}, \lambda_{3}, \ldots, \lambda_{n} \ldots$ die Nullstellen von $D(\lambda)$ sind. Somit

$$
D_{K}^{*}(\lambda)=\prod_{\nu=1}^{\infty}\left(1-\frac{\lambda}{\lambda_{\nu}}\right) e^{\frac{\lambda}{\lambda_{\nu}}}
$$

4) Wenn es nötig ist hervorzuheben, daß $D(\lambda)$ und $D^{*}(\lambda)$ mit dem Kern $K(x, y)$ gebildet sind, schreiben wir $D_{K}(\lambda)$ bzw, $D_{K}^{*}(\lambda)$. 
Hieraus ergibt sich, indem wir den reellen Teil von $\lambda_{\lambda_{\nu}}^{\lambda}$ mit $\Re\left[\begin{array}{c}\lambda^{\prime} \\ \lambda_{y}\end{array}\right]$ bezeichmen,

$$
\begin{aligned}
& \left|D_{K}^{*}(\lambda)\right|^{2}=\prod_{\nu=1}^{\infty}\left(1-2 \Re\left[\left.\begin{array}{c}
\lambda \\
\lambda_{\nu}
\end{array}|+| \begin{array}{c}
\lambda \\
\lambda_{\nu}
\end{array}\right|^{2}\right) e^{2 \Re\left[\begin{array}{c}
\lambda \\
\lambda,
\end{array}\right]}\right. \\
& \leq \prod_{y=1}^{\infty} e^{\left.-2 M\left[\begin{array}{l}
\lambda \\
\lambda_{y}
\end{array}\right]+\mid \begin{array}{c}
\lambda \\
\lambda_{y}
\end{array}\right\}^{2}+2 M\left[\begin{array}{l}
\lambda \\
\lambda_{y}
\end{array}\right]}-e^{\left.|\lambda|^{2} \Sigma^{1} \lambda_{\nu}\right|^{2}} .
\end{aligned}
$$

Weil nach einem Resultate von I. Schur")

$$
\left.\sum_{v \rightarrow 1}^{\infty} \frac{1}{\left|\lambda_{r}\right|}\right|^{2} \leqq \int_{a}^{b} \int_{a}^{t}|K(s, t)|^{2} d s d t \leqq \Omega,
$$

so ergibt sich hieraus schlieblich

$$
\left|D^{*}(\lambda)\right| \leqq e^{\frac{2}{2}|\lambda|^{2}}
$$

Dies ist, wie das folgende Beispiel lehrt, die genaue obere Grenze von $D^{*}(\lambda) \mid$ bei der Nebenbedingung (7). Schreibt man

$$
G_{m}(x, y)=e^{i \omega} \sqrt{2 m}\left\{\sum_{\nu=1}^{m} p_{r}(x) \phi_{\nu}(y)-\sum_{r=m+1}^{2 m} \phi_{\mu}(x) \phi_{m}(y)\right\},
$$

wo $\varphi_{1}(x), \varphi_{1}(x), \ldots, \varphi_{92}(x), \ldots$ ein unendliches normiertes Orthogonalsystem in bezug auf das Intervall $a \leqq x \leqq b$ ist, so wird, wenn wir zur Abkürzung $H=\dot{H} e^{i \epsilon} \sqrt{\frac{\Omega}{2 m}}$ setzen,

$$
\begin{aligned}
D_{G_{m}}^{*}(\lambda) & (1-\lambda H)^{m} e^{m \lambda H}(1+\lambda H)^{m} e^{-m \lambda H} \\
& \left(1-\lambda^{2} H^{2}\right)^{m}=\left(1-\lambda^{2} e^{2 i \theta} \frac{\Omega}{2 m}\right)^{m} .
\end{aligned}
$$

Hieraus ergibt sich, indem wir

setzen,

$$
\lambda=e^{i \eta}|\lambda|, \quad \theta+\varphi=\frac{\pi}{2}
$$

Folglich ist

$$
D_{\theta_{m}}^{*}(\lambda)=\left(1+|\lambda|^{2} \frac{\Omega}{2 m}\right)^{m} \text {. }
$$

$$
\lim _{m=\infty}\left|D_{G_{m}}^{*}(\lambda)\right|=e^{\frac{\Omega}{2}|\lambda|^{2}},
$$

woraus die Behauptung folgt.

5) Vgl. I. Schur, Uber die charalsteristisohen Warzeln einer inneren Substitution mit einer Anwendung auf die Theorie der linearen Integralgleiobungen, Math. Ann., 66 (1909), S. 488 , insb. S. 508. 
Für die Koeffizienten

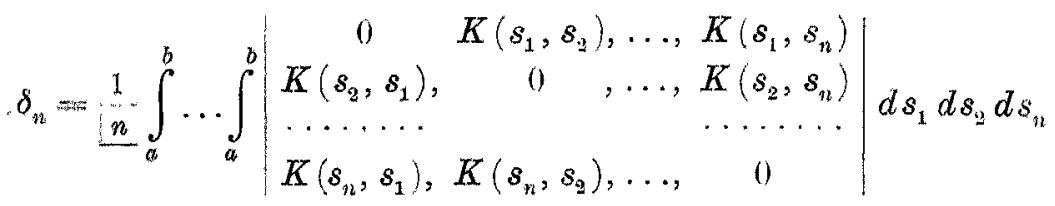

ergibt sich aus (11)

$$
\left|\delta_{n}\right| \leqq \frac{(\text { ve } \Omega)^{n}}{n^{\frac{n}{2}}} .
$$

Dies ist aber nicht die genaue obere Grenze von $\left|\delta_{n}\right|$, wie man leicht im Falle $n=2$ und $n: \cdots 3$ findet.

Das Problem, das Maximum von $\left|D^{*}(\lambda)\right|$ und $\delta_{1}$ zu finden, läßBt sich mit den gewöbnlichen Methoden der Variationsrechnung behandeln. Ich hoffe, auf diese Frage in einer anderen Arbeit zurückzukommen.

Wir gehen nun dazu :über, $D^{*}\left(\begin{array}{l}x \\ y\end{array} \mid \lambda\right)$ abzuschätzen und betrachten dabei zunächst reelle symmetrische Kerne $K(x, y)$. Es ist, indem wir unter $\lambda_{1}, \lambda_{2}, \ldots, \lambda_{n}, \ldots$ die Eigenwerte und unter $\phi_{1}, \phi_{2}, \ldots, \phi_{n}, \ldots$ die zugehörigen normierten Eigenfunktionen verstehen,

$$
\begin{aligned}
& D^{*}\left(\begin{array}{l}
x \\
y
\end{array} \mid \lambda\right)=D^{*}(\lambda)\left(K(x, y)+\lambda \frac{\sum \gamma \varphi_{n}(x) \varphi_{n}(y)}{\lambda_{n}\left(\lambda_{\mu}-\lambda\right)}\right) \\
& =D^{*}(\lambda) K(x, y)+\lambda \frac{\sum}{n} \frac{p_{n}(x)}{\lambda_{n}} p_{n}(y) I^{*}(\lambda) .
\end{aligned}
$$

Ferner gilt

$$
\begin{aligned}
& \frac{D^{*}(\lambda)}{1-\frac{\lambda}{\lambda_{n}}}=e^{\frac{\lambda}{\lambda_{n}}} \prod_{\nu \neq n}\left(1-\frac{\lambda}{\lambda_{\nu}}\right) e^{\lambda_{\nu}}
\end{aligned}
$$

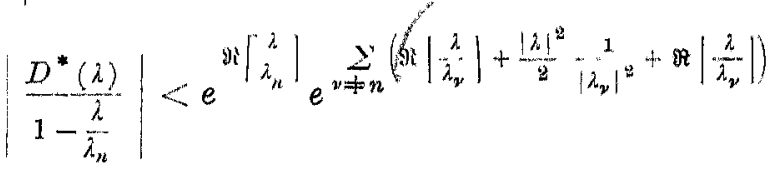

$$
\begin{aligned}
& =e^{\left.\Re i \frac{\lambda}{\lambda}\right]} e^{\frac{|\lambda|^{2}}{2} y \neq n\left|\lambda_{\nu}\right|^{2}}<e^{|\lambda| \sqrt{\alpha}+|\lambda|^{2} \Omega}
\end{aligned}
$$

Somit

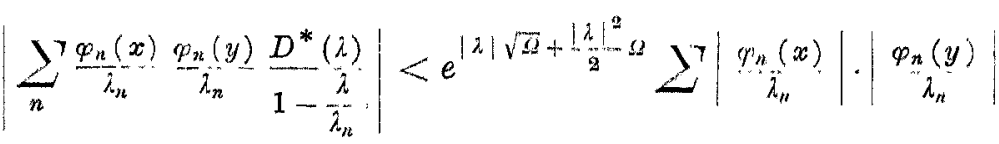

$$
\begin{aligned}
& \leqq e^{\frac{|\lambda|^{2}}{2} a+|\lambda| \sqrt{x}} V \int_{a}^{b} K(x, s)^{2} d s \cdot \int_{a}^{b} K(y, s)^{2} d s
\end{aligned}
$$


Wir bekommen also schließlich die folgende Ungleichung

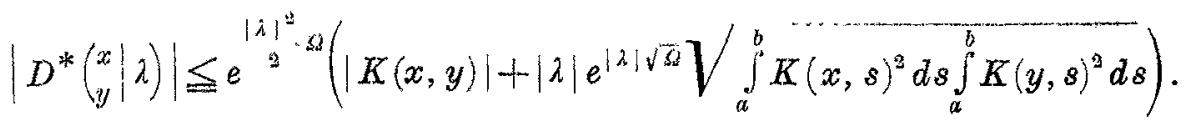

Um im allgemeinen Falle, wo $K(x, y)$ eine komplexe unsymmetrische Funktion ist, eine Relation vop der Form (13) zu erhalten, beweisen wir zunächst die folgende Determinantenungleichung. Es sei

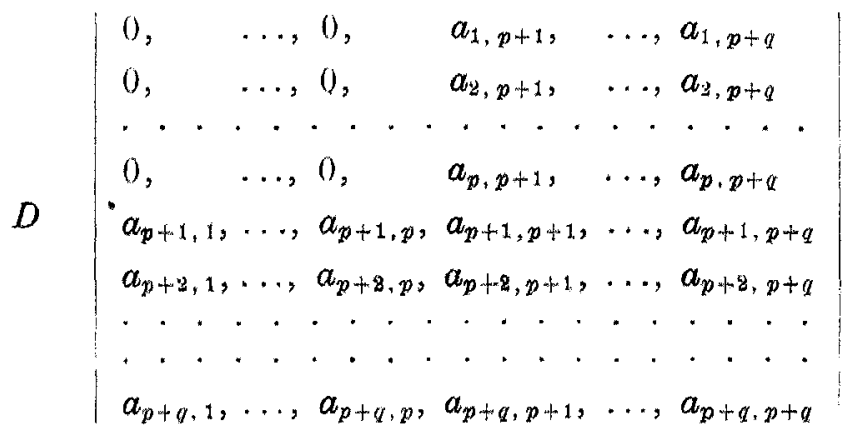

eine Determinante, wo $q \cdot p$, und ferner

$$
\begin{aligned}
& \sum_{n, p=1}^{q}\left|a_{n, p \mid p}\right|^{2}=L_{n} \quad(n=1,2, \ldots, p), \\
& \sum_{1}^{\prime \prime}\left|a_{p+n}, n\right|^{2}=M_{n} \quad(n=1,2, \ldots, p), \\
& \sum_{s=1}^{7} \sum_{t}^{y}\left|a_{p \mid s, p+t}\right|^{2}-N \text {. }
\end{aligned}
$$

Dann gilt

$$
|D| \leqq\left(L_{1} L_{\mathrm{z} 2} \ldots L_{p}\right)^{\frac{1}{2}} \cdot\left(M_{1} M_{2} \ldots M_{p}\right)^{\frac{1}{2}}-\frac{N^{q-p}}{(q-p)^{2}}
$$

Das Gleichheitszeichen kann nicht ausgeschlossen werden.

Der von mir ursprünglich gefundene Beweis für diesen Determinantensatz macht von den Hilfsmitteln der Differentialrechnung Gebrauch. Herrn M. Riesz, dem ich den Satz mitgeteilt habe, verdanke ich einen etwas kürzeren Beweis, der ebenfalls eine der Infinitesimalrechnung angehörende Betrachtung benutzt. Ich lasse hier einen rein algebraischen Beweis folgen, auf den mich Herr I. Schur aufmerksam gemacht hat:

Durch eine unitär orthogonale lineare Transformation der $q$ letzten Zeilen und eine ebensolche Transformation der $q$ letzten Kolonnen kann, wie in bekannter Weise leicht geschlossen wird, erreicht werden, daß $D$ in eine Determinante $D^{\prime}=\left|a_{\varkappa \lambda}^{\prime}\right|$ übergeht, in der nicht nur für $x \leqq p$, 
$\lambda \leqq p$, sondern auch für $x \leqq p, \lambda>p+\varkappa$ oder $\lambda \leqq p, x>p+\lambda$ die Elemente $a_{\% \lambda}^{\prime}$ den Wert Null erhalten. Hierbei bleiben die Zahlen $|D|$, $L_{n}, M_{n}$ und $N$ ungeändert. Es wird aber

$$
D^{\prime}=(-1)^{p} a_{1, p+1}^{\prime} a_{2, p+2}^{\prime} \ldots a_{p, 2 p}^{\prime} a_{p+1,1}^{\prime} a_{p+2,2}^{\prime} \ldots a_{2 p, p}^{\prime}\left|\begin{array}{c}
a_{2 p+1,2 p+1}^{\prime}, \ldots, a_{2 p+1, p+q}^{\prime} \\
\cdot . \ldots . . . \\
a_{p+q, 2 p+1}^{\prime}, \ldots, a_{p+q, p+q}^{\prime}
\end{array}\right| .
$$

Nach dem Hadamardschen Determinantensatz wird daher

$$
|D|^{2}=\left|D^{\prime}\right|^{2} \leqq \prod_{x=1}^{p} a_{x, p+x}^{\prime} a_{p+x, x}^{\prime}, \prod_{\alpha=1}^{q-p} \sum_{\beta=1}^{q-p} \mid a_{2 p+\alpha, 2 p+\left.\beta\right|^{\prime}}^{\prime}
$$

Hieraus folgt die zu beweisende Ungleichung (18), indem man beachtet, daB

$$
\left|a_{\varkappa, p+x}^{\prime}\right|^{2} \leqq \sum_{\lambda=1}^{q}\left|a_{x, p+\lambda}^{\prime}\right|^{2}=L_{\varkappa}, \quad\left|a_{p+\varkappa, x}^{\prime}\right|^{2} \leqq \sum_{\lambda=1}^{u}\left|a_{p+\lambda, x}^{\prime}\right|^{2} * M_{x}
$$

und nach dem Satz vom arithmetischen und geometrischen Mittel

wird.

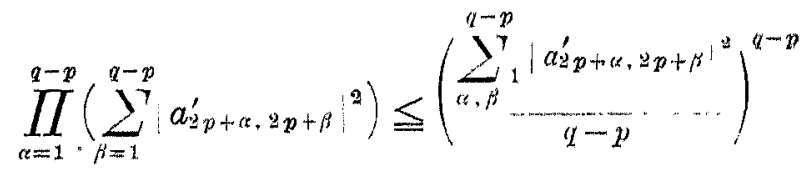

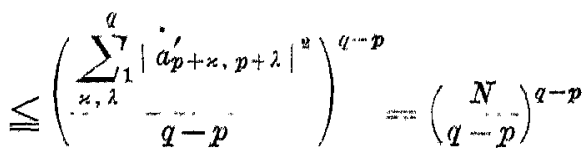

Wir kehren jetzt zu unserer Aufgabe zurück, eine obere Grenze von $\left|D^{*}\left(\begin{array}{l}x \\ y\end{array} \mid \lambda\right)\right|$ zu finden. Dabei werden die folgenden Formeln benutzt, die aus den Resultaten von Hilbert (Grundzüge einer allgemeinen Theorie der linearen Integralgleichungen, Kap. I) leicht gefolgert werden können. Es sei

$$
\begin{gathered}
k_{p, q}^{(n)}=K\left(a+\frac{l p}{n}, a+\frac{l q}{n}\right) \\
k_{x, p}^{(n)}=K\left(x, a+\frac{l p}{n}\right), \quad k_{p, y}^{(n)}=K\left(a+\frac{l_{p} p}{n}, y\right) .
\end{gathered}
$$

Dann gelten die Beziehungen:

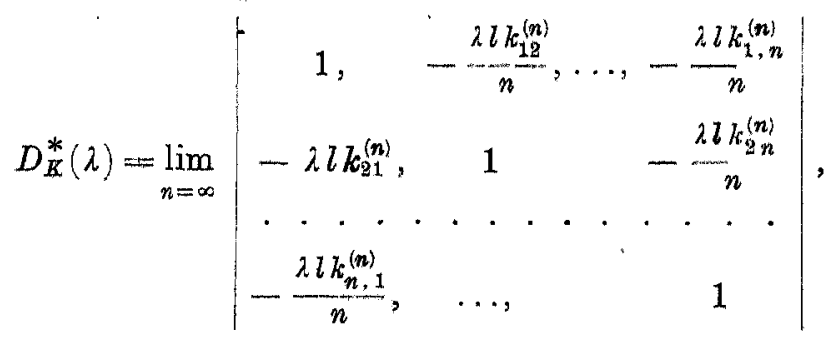


(20) $D_{K^{*}}^{*}\left(\begin{array}{l}x \\ y\end{array} \mid \lambda\right)=D_{K}^{*}(\lambda) K(x, y)$

$+\lambda \lim _{n \rightarrow \infty}$

$$
\begin{aligned}
& 0, \quad-\cdots \sqrt{l} k_{x, 1}^{(n)}, \cdots \sqrt{\frac{l}{n}} k_{x, y}^{(n)}, \ldots,-\sqrt{\frac{l}{n}} k_{x, n}^{(n)} \\
& \sqrt{\frac{l}{n}} k_{1, y}^{(n)}, \quad 1, \quad-\frac{\lambda l}{n} k_{12}^{(n)}, \quad \ldots,-\frac{\lambda l}{n} k_{1, n}^{(n)} \\
& \sqrt{h} k_{n, y,}^{\langle n\rangle},-\frac{\lambda l}{n} k_{n !}^{(n)}, \quad 1, \quad \ldots,-\frac{\lambda l}{n} k_{3, n}^{(n)} \\
& V_{n}^{l} k_{n, y}^{(n)},-\sum_{n}^{\lambda l} k_{n, 1}^{(n)}, \quad \ldots, \quad 1,
\end{aligned}
$$

Infolge der Hadamardschen Ungleichung ergibt sich

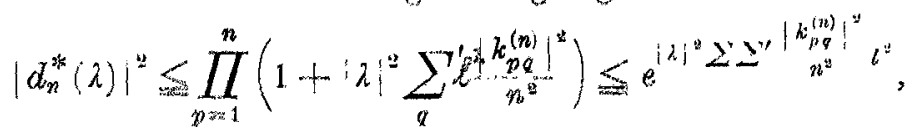

woraus durch' einen Grenzübergang die Relation (11) folgt. Wenden wir auf die in (20) auftretende Determinante den oben erwähnten Determinantensatz $(p \ldots 1, q \ldots n)$ an, so finden wir

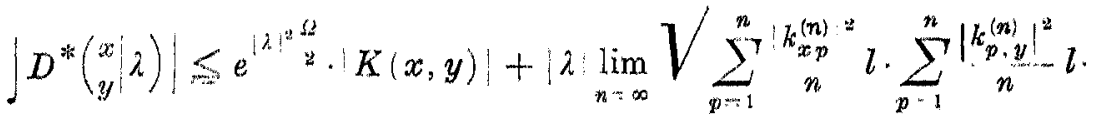

$$
\begin{aligned}
& \cdot \lim _{n \rightarrow \infty}\left\{\left(n+\sum_{p, q}^{\gamma^{\prime}|\lambda|^{2} l^{3}}\left|k_{p q}^{(n)}\right|^{2}\right)^{\frac{n-1}{2}} !{ }_{(n-1)^{\frac{n-1}{2}}}\right\} \cdots
\end{aligned}
$$

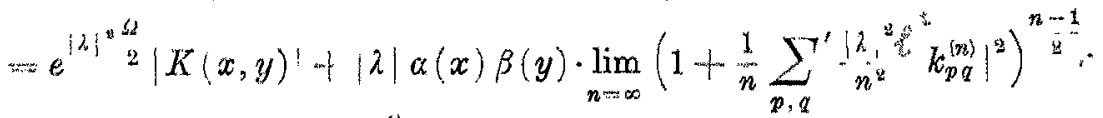

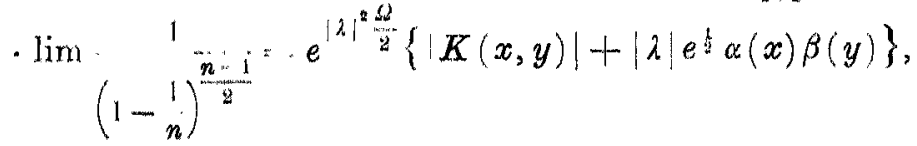

wo

$$
a(x)^{2}=\int_{a}^{b}|K(x, s)|^{2} d s, \quad \beta(y)^{2}=\int_{a}^{b}|K(s, y)|^{2} d s
$$

gesetzt ist. Es hat sich somit ergeben

$$
\left|D^{*}\left(\begin{array}{l}
x \\
y \mid
\end{array} \mid \lambda\right)\right| \leq e^{|\lambda|^{2} \frac{\Delta}{y}}\left\{|K(x, y)|+\mid \lambda^{\prime} \sqrt{e} a(x) \beta(y)\right\} .
$$

\section{$\S 2$.}

In diesem Paragraphen untersuchen wir die Reihen $D^{*}(\lambda)$ und $D^{*}\left(\begin{array}{l}x \\ y\end{array} \mid \lambda\right)$ unter der Voraussetzung, daß $K(s, t)$ eine solche meßbare Funktion ist, für welche

$$
\int_{a}^{b} \int_{a}^{b}|K(s, t)|^{2} d s d t
$$


existiert. Wir müssen einerseits die Existenz der in den Koeffizienten von $D^{*}(\lambda)$ und $D^{*}\left(\begin{array}{l}x \\ y\end{array} \mid \lambda\right)$ auftretenden mehrfachen Integrale beweisen, andererseits zeigen, daß diese Reihen ganze Funktionen von $\lambda$ darstellen. Dies gelingt durch einen Grenzübergang unter Verwendung der im vorigen Paragraphen gewonnenen Resultate.

Es sei $\Phi_{1}(x, y), \Phi_{2}(x, y), \ldots, \Phi_{n}(x, y), \ldots$ eine Folge stetiger Funktionen, die (in bezug auf das Gebiet $a \leqq_{y}^{x} \leqq b$ ) ein vollständiges normiertes Orthogonalsystem bilden. Schreiben wir

$$
C_{p}=\int_{a}^{b} \int_{a}^{b} K(s, t) \Phi_{p}(s, t) d s d t
$$

so läßt $\operatorname{sich}^{\theta}$ ) eine Indexfolge $m_{1}, m_{2}, \ldots, m_{n} \ldots$ so auswählen, daß

$$
G_{p^{\prime}}(x, y)=\sum_{p=1}^{m_{y^{\prime}}} C_{p} \Phi_{p}(x, y)
$$

für $\nu \rightarrow \infty$ fast überall gegen $K(x, y)$ konvergiert. Diese Funktionenfolge besitzt noch die Eigenschaften:

$$
\begin{gathered}
\int_{a}^{b} \int_{a}^{b}\left|G_{\nu}(x, y)\right|^{2} d y \leqq \int_{a}^{b} \int_{a}^{b}|K(x, y)|^{2} d x d y, \\
\lim _{\nu=\infty} \int_{a}^{b} \int_{a}^{b}\left|K(x, y)-G_{\nu}(x, y)\right|^{2} d x d y=0 .
\end{gathered}
$$

Die $G_{\nu}(x, y)$ können ferner, was für das Folgende wichtig ist, so gewählt werden, daß die Relationen

$$
\left\{\begin{array}{l}
\lim _{\nu=\infty} \int_{a}^{b}\left|K(x, s)-G_{v}(x, s)\right|^{2} d s=0 \\
\lim _{v=\infty} \int_{a}^{b}\left|K(s, y)-G_{v}(s, y)\right|^{2} d s=0
\end{array}\right.
$$

für fast alle $x$ - und $y$-Werte im Intervalle $(a, b)$ erfüllt sind. Dies ergibt sich unmittelbar aus dem folgenden Satze: Es seien

$$
\Omega_{1}(x, y), \Omega_{2}(x, y), \ldots, \Omega_{n}(x, y), \ldots
$$

für fast alle Werte im Gebiete $a \leqq_{y}^{x} \leqq b$ definierte Funktionen, die positiv und summierbar sind und ferner noch die Bedingung

erfüllen.

$$
\lim _{n=\infty} \int_{a}^{b} \int_{a}^{b} \Omega_{n}(x, y) d x d y=0
$$

๑) Vgl. H. Weyl, Math. Ann. 67 (1909), S. $225-245$ insb. S. 243. 
Dann kann man eine Indexfolge $n_{1}, n_{\sharp}, \ldots, n_{v}, \ldots$ so auswählen, daß für fast alle $x$-Werte $(a \leqq x \leqq b)$

$$
\left.\lim _{y \rightarrow-\infty} \int_{a}^{b} \Omega_{n_{\nu}}(x, y) d y=0^{i}\right)
$$

ist. Zum Beweise schreiben wir

$$
\int_{a}^{b} \int_{a}^{b} \Omega_{n}(x, y) d x d y=c_{n}, \quad f_{n}(x)=\int_{a}^{b} \Omega_{n} \cdot(x, y) d y
$$

und wählen die Indexfolge $n_{1}, n_{2}, \ldots, n_{\nu}, \ldots$ so, daB $\sum_{p=1}^{\infty} \alpha_{n_{\nu}}$ konvergiert. Es seien $\varepsilon_{1}, \varepsilon_{2}, \ldots, g_{n}, \ldots$ positive gegen Null abnehmende Zahlen. Wir bezeichnen mit $E_{p}$ diejenige Punktmenge im Intervalle $a \leqq x \leqq b$, für welche

$$
\lim _{v \rightarrow \infty} f_{n_{\nu}}(x) \geqq \varepsilon_{p} \text {. }
$$

Alle $x$-Werte, die so beschaffen sind, daß $\lim _{x \rightarrow \infty} f_{n_{\nu}}(x)$ nicht existiert oder nicht gleich Null ist, sind in der Menge

$$
E_{1}+E_{0}+E_{3}+\ldots+E_{p}+\ldots
$$

enthalten. Hieraus folgt unser Satz, falls wir noch beweisen, daß jede Punktmenge $E_{p}$ vom Maße Null ist. Dies folgt aber leicht so: Das Maß derjenigen Punktmenge $H(p, v)$, für welche

$$
f_{n, p}(x) \geqq \underset{2}{s_{p}}
$$

ist wegen

kleiner als

$$
\int_{a}^{b} f_{n_{\nu}}(x) d x=a_{n_{y}}
$$

Weil nun $E_{p}$ sicher der Menge

$$
2 \alpha_{n_{\nu}}
$$

$$
\sum_{v: m m}^{\infty} H(p, v)
$$

wo $m$ beliebig groß sein kann, angehört, so ist das Maß von $E_{p}$ kleiner als

$$
\frac{2}{\varepsilon_{v}} \sum_{\nu=m}^{\infty} \alpha_{n_{\nu}}
$$

welche Größe wegen der Konvergenz von $\sum \alpha_{n_{v}}$ für genügend große $m$ kleiner als jede vorgegebene positive Zahl wird. Jede Menge $E_{p}$ ist also vom Maße Null und folglich auch $\sum E_{p}$, w. z. b. w.

7) Nach einem Satz der Lesbosguesohen Integration von Doppelintegralen existiert dies Integral für fast alle $x$ im Intervalle $a \leqq x \leqq b$. 
Die Meßbarkeit von $K(s, t)$ hat zur Folge, daß auch

$$
\dot{K}\left(s_{1}, s_{2}\right) K\left(s_{2}, s_{z}\right) \ldots \ldots K\left(s_{n-1}, s_{n}\right) K\left(s_{n}, s_{1}\right)
$$

im zugehörigen $n$-dimensionalen Gebiete $\left(D_{n}\right)$, meßbar ist. Um einzusehen, daß (25) in $D_{n}$ auch summierbar ist, brauchen wir den folgenden Satz $\left.^{8}\right)$ : „Wenn eine Folge von nicht negativen summierbaren Funktionen

$$
f_{1}\left(x_{1}, x_{0}, \ldots, x_{n}\right), \quad f_{2}\left(x_{1}, x_{2}, \ldots, x_{n}\right), \ldots, f_{n}\left(x_{1}, x_{2}, \ldots, x_{n}\right), \ldots
$$

fast überall gegen eine Grenzfunktion $f\left(x_{1}, x_{2}, \ldots, x_{n}\right)$ konvergiert und wenn es ferner eine solche von $n$ unabhängige Konstante $K$ gibt, daß

$$
\iint \ldots \int f_{n}\left(x_{1}, x_{2}, \ldots, x_{n}\right) d x_{1} d x_{2} \ldots d x_{n}, \ldots K
$$

so ist $f\left(x_{1}, x_{2}, \ldots, x_{n}\right)$ summierbar und

$$
\iint \ldots \int f\left(x_{1}, x_{2}, \ldots, x_{n}\right) d x_{1} d x_{2} \ldots d x_{n} \leqslant K^{\prime} .
$$

Man findet, wie folgt, eine obere Grenze von

$$
\int_{a}^{b} \int_{a}^{b} \ldots \int_{a}^{b}\left|G_{m}\left(s_{1}, s_{2}\right)\right| \cdot\left|G_{m}\left(s_{2}, s_{3}\right)\right| \ldots\left|G_{m}\left(s_{n}, s_{1}\right)\right| d s_{1} d s_{2} \ldots d s_{12} .
$$

Aus der Schwarzschen Ungleichung ergibt sich, indem wir die Bezeichnungen

einführen,

$$
\begin{aligned}
& h(x)^{2}=\int_{a}^{b}\left|G_{m}(x, y)\right|^{2} d y, \\
& k(x)^{2}=\int_{a}^{b}\left|G_{m}(y, x)\right|^{2} d y
\end{aligned}
$$

$$
\int_{a}^{b}\left|G_{m}\left(s_{1}, s_{2}\right)\right| \cdot\left|G_{m}\left(s_{2}, s_{3}\right)\right| d s_{2} \leqq h\left(s_{1}\right) k\left(s_{2}\right)
$$

Ferner

$$
\begin{aligned}
& \int_{a}^{b} \int_{a}^{b}\left|G_{m}\left(s_{1}, s_{3}\right)\right| \cdot\left|G_{m}\left(s_{2}, s_{3}\right)\right| \cdot\left|G_{m}\left(s_{3}, s_{4}\right)\right| d s_{2} d s_{3} \\
\leqq & \int_{a}^{b} h\left(s_{1}\right) k\left(s_{3}\right) G_{m}\left(s_{3}, s_{4}\right) \mid d s_{3} \\
\leqq & h\left(s_{1}\right) \sqrt{\int_{a}^{b} k\left(s_{3}\right)^{2}} d s \cdot k\left(s_{4}\right)=h\left(s_{1}\right) \sqrt{\int_{a}^{b} \int_{a}^{b}\left|G_{m}(s, t)\right| d s d t k\left(s_{4}\right) .}
\end{aligned}
$$

Setzen wir hier $s_{1}=s_{1}$, so ergibt sich nach Integration und nochmaliger Anwendung der Schwarzschen Ungleichung

$\int_{a}^{b} \int_{a}^{b} \int_{a}^{b}\left|G_{m}\left(s_{1}, s_{2}\right) G_{m}\left(s_{2}, s_{3}\right) G_{m}\left(s_{3}, s_{1}\right)\right| d s_{1} d s_{2} d s_{3} \leqq\left(\sqrt{\int_{a}^{b} \int_{a}^{b}\left|G_{m}(s, t)\right|^{2} d s d t}\right)^{3}$.

8) Vgl. Fatou, Séries trigonometriques et séries de Taylor (Acta matematica 30 (1906), S. 375). 
Fahren wir in derselben Weise fort, so erhalten wir für ein beliebiges $n$

$$
\begin{gathered}
\int_{a}^{b} \int_{a}^{b} \ldots \int_{a}^{b}\left|G_{m}\left(s_{1}, s_{2}\right) G_{m}\left(s_{2}, s_{3}\right) \ldots G_{m}\left(s_{n}, s_{1}\right)\right| d s_{1} d s_{2} \ldots d s_{n} \\
\leqq\left[\left.\int_{a}^{b}\left|\int_{a}^{b}\right| G_{n}(s, t)\right|^{2} d s d t\right]^{n} \leqq \Omega^{2} .
\end{gathered}
$$

Beriicksichtigt man noch, daß fast überall im Gebiete $D_{n}$

$$
\lim _{n}\left|G_{m}\left(s_{1}, s_{2}\right) \ldots G_{m}\left(s_{n}, s_{1}\right)\right|=\left|K\left(s_{1}, s_{2}\right) \ldots K\left(s_{n}, s_{1}\right)\right| \text {, }
$$

so ergibt sich aus dern oben erwähnten Satz, daß

und a fortiori

$$
\left|K\left(s_{1}, s_{2}\right) K\left(s_{2}, s_{2}\right) \ldots K\left(s_{n}, s_{1}\right)\right|
$$

$$
K\left(s_{1}, s_{2}\right) K\left(s_{2}, s_{3}\right) \ldots K\left(s_{n}, s_{1}\right)
$$

in $D_{1}$ summierbar ist. Weil nun jedes Glied in der Determinante

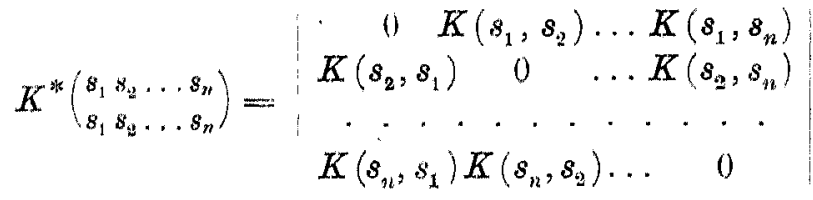

ein Produkt von Faktoren ist, die alle die Form (25) haben und von denen nie zwei gemeinsame Veränderlichen enthalten, so folgt hieraus die Existenz der Integrale

$$
\int_{a}^{b} \int_{a}^{b} \ldots \int_{a}^{b} K^{*}\left(\begin{array}{c}
s_{1} s_{2} \ldots s_{n} \\
s_{1} s_{3} \ldots s_{n}
\end{array}\right) d s_{1} d s_{2} \ldots d s_{n} .
$$

Aus der Identität

$$
\begin{aligned}
& K\left(s_{1}, s_{2}\right) K\left(s_{3}, s_{3}\right) \ldots K\left(s_{n}, s_{1}\right)-G_{m}\left(s_{1}, s_{3}\right) G_{m}\left(s_{2}, s_{3}\right) \ldots G_{m}\left(s_{n}, s_{1}\right) \\
& =:\left(K\left(s_{1}, s_{2}\right)-G_{m}\left(s_{1}, s_{2}\right)\right) K\left(s_{2}, s_{3}\right) \ldots K\left(s_{n}, s_{1}\right) \\
& +G_{m b}\left(s_{1}, s_{2}\right)\left[K\left(s_{21}, s_{3}\right)-G_{m}\left(s_{2}, s_{3}\right)\right] K\left(s_{3}, s_{1}\right) \ldots K\left(s_{n}, s_{1}\right) \\
& +G_{m k}\left(s_{1}, s_{2}\right) G_{m}\left(s_{2}, s_{3}\right)\left[K\left(s_{3}, s_{4}\right)-G_{m}\left(s_{3}, s_{4}\right)\right] K\left(s_{1}, s_{5}\right) \ldots K\left(s_{n}, s_{1}\right) \\
& +G_{m}\left(s_{1}, s_{2}\right) G_{m}\left(s_{2}, s_{3}\right) \ldots\left[K\left(s_{n}, s_{1}\right)-G_{m}\left(s_{n}, s_{1}\right)\right]
\end{aligned}
$$

erhält man durch Anwendung der Schwarzschen Ungleichung unter Berücksichtigung der Gleichung (23), daß

$$
\begin{gathered}
\int_{a}^{b} \int_{a}^{b} \ldots \int_{i b}^{b} K\left(s_{1}, s_{2}\right) K\left(s_{2}, s_{3}\right) \ldots K\left(s_{n}, s_{1}\right) d s_{1} d s_{2} \ldots d s_{n} \\
=\lim _{m=\infty} \int_{a}^{b} \int_{a}^{b} \ldots \int_{t}^{b} G_{m}\left(s_{1}, s_{2}\right) G_{m}\left(s_{2}, s_{8}\right) \ldots G_{m}\left(s_{n}, s_{1}\right) d s_{1} d s_{2} \ldots d s_{n}
\end{gathered}
$$


und somit

$$
\begin{aligned}
& \lim \int_{a}^{b} \int_{a}^{b} \ldots \int_{a}^{b} G_{m}^{*}\left(\begin{array}{c}
s_{1} s_{2} \ldots s_{n} \\
s_{1} s_{2} \ldots s_{n}
\end{array}\right) d s_{1} d s_{2} \ldots d s_{n} \\
& =\int_{a}^{b} \int_{a}^{b} \ldots \int_{a}^{b} K^{*}\left(\begin{array}{c}
s_{1} s_{2} \ldots s_{n} \\
s_{1} s_{2} \ldots s_{n}
\end{array}\right) d s_{1} d s_{2} \ldots d s_{n} .
\end{aligned}
$$

Weil die Ungleichung (12) für jede der Größen

$$
\left|\frac{1}{1 n} \int_{a}^{b} \int_{a}^{b} \ldots \int_{a}^{b} G_{m}^{*}\left(\begin{array}{c}
s_{1} s_{2} \ldots s_{n} \\
s_{1} s_{2} \ldots s_{n}
\end{array}\right) d s_{1} d s_{2} \ldots d s_{n}\right|
$$

gilt, so folgt ihre Gültigkeit auch für

$$
\left|\frac{1}{\lfloor n} \int_{a}^{b} \int_{a}^{b} \ldots \int_{a}^{b} K^{*}\left(\begin{array}{c}
s_{1} s_{2} \ldots s_{n} \\
s_{1} s_{2} \ldots s_{n}
\end{array}\right) d s_{1} d s_{22} \ldots d s_{n}\right| .
$$

Also ist $D_{K}^{*}(\lambda)$ eine ganze Funktion von $\lambda$. Ferner ergibt sich leicht

Mithin

$$
\lim _{m=\infty} D_{G_{m}}^{*}(\lambda)=D_{K}^{*}(\lambda)
$$

$$
\left|D_{\mathbb{K}}^{*}(\lambda)\right| \leqq e^{\frac{1}{2}|\lambda|^{2} \Omega}
$$

Man kann nun $D^{*}\left(\begin{array}{l}x \\ y\end{array} \mid \lambda\right)$ in ganz analoger Weise untersuchen. Zunächst wird mit Hilfe von (22), (23) und (24) bewiesen, daß

$$
\int_{a}^{b} \int_{a}^{b} \ldots \int_{a}^{b} K\left(x, s_{1}\right) K\left(s_{1}, s_{2}\right) \ldots K\left(s_{n}, y\right) d s_{1} d s_{2} \ldots d s_{n} \ldots K^{(n+1)}(x, y)
$$

für fast alle $x, y$-Werte $\left(a \leqq_{y}^{x} \leqq b\right)$ existiert und ferner, daß

$$
\lim _{m=\infty} G_{m}^{(p)}(x, y)=K^{(p)}(x, y) \text {. }
$$

Hieraus folgt mit Rücksicht auf (26), daß (mit Ausnahme von höchstens einer Nullmenge) die Integrale in den Koeffizienten von $D_{K}^{*}\left(\begin{array}{l}x \\ y\end{array} \mid \lambda\right)$ für $m \rightarrow \infty$ gegen die entsprechenden der Reihe $D_{\mathbb{K}}^{*}\left(\begin{array}{l}x \\ y\end{array} \mid \lambda\right)$ konvergieren. Wegen (21), angewendet auf $G_{m}(x, y)$, bleibt $D_{G_{m}}^{*}\left(\begin{array}{l}x \\ y\end{array} \mid \lambda\right)$ kleiner als eine von $m$ unabhängige nur von $x, y, \lambda$ abhängige Konstante. Aus diesen beiden Tatsachen folgt nach einem bekannten Satze, daß $D_{\boldsymbol{K}}^{*}\left(\begin{array}{l}x \\ y\end{array} \mid \lambda\right)$ eine überall konvergente Potenzreihe von $\lambda$ ist, die als Grenzwert von $D_{G_{m}}^{*}\left(\begin{array}{l}x \\ y\end{array} \mid \lambda\right)$ für $m \rightarrow \infty$ dargestellt werden kann. Ferner genügt $D_{K}^{*}\left(\begin{array}{l}x \\ y\end{array} \mid \lambda\right)$ mit Ausnabme 
von höchstens einer Nullmenge der Ungleichung (21), woraus folgt, daß $D_{K}^{*}\left(\begin{array}{l}x \\ y\end{array} \mid \lambda\right)$ und $D_{K}^{*}\left(\begin{array}{l}x \\ y\end{array} \mid \lambda\right)^{2}$ im Gebiete $a \leqq_{y}^{x} \leqq b$ summierbare Funktionen sind.

Wir beweisen noch, daß für fast alle $x$ und $y$ die Relationen

$$
\begin{aligned}
& \lim _{m \rightarrow \infty} \int_{a}^{b}\left|D_{G_{m}}^{*}\left(\begin{array}{l}
x \\
s
\end{array} \mid \lambda\right)-D_{K}^{*}\left(\begin{array}{l}
x \\
s
\end{array} \mid \lambda\right)\right|^{2} d s=0, \\
& \lim _{m \rightarrow=\infty} \int_{a}^{b}\left|D_{G_{m}}^{*}\left(\begin{array}{l}
s \\
y
\end{array} \mid \lambda\right)-D_{K}^{*}\left(\begin{array}{l}
s \\
y
\end{array} \mid \lambda\right)\right|^{2} d s=0
\end{aligned}
$$

gelten. Es möge für die Summe der ersten $p$ Glieder einer Potenzreihe $P(\lambda)$ die Bezeichnung $\{P(\lambda)\}_{p}$ eingeführt werden. Die Summe der übrigbleibenden Glieder nennen wir $\{P(\lambda)\}^{(p)}$. Es ist

$$
\int_{a}^{b}\left|D_{x_{m}}^{*}\left(\begin{array}{l}
x \\
s
\end{array} \mid \lambda\right)-D_{K}^{*}\left(\begin{array}{l}
x \\
s
\end{array}\right)\right|^{2} d s
$$

$\leqq 2 \int_{a}^{b}\left\{\left.\left\{D_{G_{m}}^{*}\left(\begin{array}{l}x \\ s\end{array} \mid \lambda\right)-D_{K}^{*}\left(\begin{array}{l}x \\ s\end{array} \mid \lambda\right)\right\}_{p}\right|^{2} d s+2 \int_{a}^{b}\left|\left\{D_{a_{m}}^{*}\left(\begin{array}{l}x \\ s\end{array} \mid \lambda\right)-D_{K}^{*}\left(\begin{array}{l}x \\ s\end{array} \mid \lambda\right)\right\}^{(p)}\right|^{2} d s\right.$.

Wegen dex leicht zu beweisenden Relation

$$
\lim _{m \rightarrow \infty} \int_{a}^{b}\left|K^{(p)}(x, s)-G_{m}^{(p)}(x, s)\right|^{2} d s=0 \quad \text { (für fast alle } x \text { ) }
$$

haben wir fast überall

$$
\lim _{m=\infty} \int_{a}^{b}\left|\left\{D_{G_{m}}^{*}\left(\begin{array}{c}
x \\
s
\end{array} \mid \lambda\right)-D_{K}^{*}\left(\begin{array}{l}
x \\
s
\end{array} \mid \lambda\right)\right\}_{p}\right|^{2} d s=0
$$

Sei nun $|\lambda|<R$, wo $R$ eine beliebige positive Zahl ist, ferner $C_{2 R}$ ein Kreis mit dem Radius $2 R$ um den Nullpunkt.

Wir haben

Folglich wegen (21)

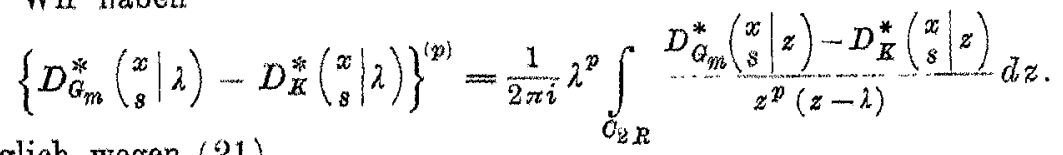

$$
\begin{aligned}
\mid\left\{D_{G_{m}}^{*}\left(\begin{array}{l}
x \\
s
\end{array} \mid \lambda\right)\right. & \left.-D_{K}^{*}\left(\begin{array}{l}
x \\
s
\end{array} \mid \lambda\right)\right\}^{(p)} \mid \leq 2 \cdot\left(\begin{array}{l}
1 \\
2
\end{array}\right)^{p} e^{2 R^{2} s}\left[|K(x, s)|+\left|G_{m}(x, s)\right|\right. \\
& +2 R \sqrt{e} \sqrt{\int_{a}^{b}|K(x, t)|^{2} d t \cdot \int_{a}^{b}|K| t,\left.s\right|^{2} d t} \\
& +2 R \sqrt{e} \sqrt{\left.\int_{a}^{b}\left|G_{m}(x, t)\right|^{2} d t \cdot \int_{a}^{b}\left|G_{m}(t, s)\right|^{2} d t\right] .}
\end{aligned}
$$


Erheben wir beide Seiten dieser Ungleichung ins Quadrat und integrieren in bezug auf $s$, so erkennen wir auf Grund der Relationen (22) und (24), daß

$$
\int_{a}^{b}\left\{\left\{D_{G_{m}}^{*}\left(\begin{array}{l}
x \\
s
\end{array} \mid \lambda\right)-D_{K}^{*}\left(\begin{array}{l}
x \\
s
\end{array} \mid \lambda\right)\right\}^{|p|} \quad d s \leqq\left(\frac{1}{2}\right)^{p} C(x, y),\right.
$$

wo $C(x, y)$ eine für fast alle $x$ endliche Größe bedeutet, die von $p$ und $m$ urabhängig ist. Aus (29), (30) und (31) ergibt sich nun leicht (27). Auf analoge. Weise erhält man (28).

Wir haben nun die nötigen Hilfsmittel gewonnen, um aus den Resolventengleichungen

$$
\begin{aligned}
& D_{\alpha_{m}}^{*}\left(\begin{array}{l}
x \\
y
\end{array} \mid \lambda\right)-\lambda \int_{a}^{b} G_{m}(x, s) D_{G_{m}}^{*}\left(\begin{array}{l}
s \\
y
\end{array} \mid \lambda\right) d s=D_{j_{m}}^{*}(\lambda) G_{m}(x, y), \\
& D_{x_{m}}^{*}\left(\begin{array}{l}
x \\
y
\end{array} \mid \lambda\right)-\lambda \int_{a}^{b} D_{a_{m}}^{*}\left(\begin{array}{l}
x \\
s
\end{array} \mid \lambda\right) G_{m}(s, y) d s=D_{z}^{*}(\lambda) G_{m}(x, y)
\end{aligned}
$$

durch einen Grenzübergang $(m \rightarrow \infty)$ die Relationen (fast überall)

$$
\left\{\begin{array}{l}
D_{K}^{*}\left(\begin{array}{l}
x \\
y
\end{array} \mid \lambda\right)-\lambda \int_{a}^{b} K(x, s) D_{K}^{*}\left(\begin{array}{l}
s \\
y
\end{array} \mid \lambda\right) d s=D^{*}(\lambda) K(x, y), \\
D_{K}^{*}\left(\begin{array}{c}
x \\
y
\end{array} \mid \lambda\right)-\lambda \int_{a}^{b} D_{K}^{*}\left(\begin{array}{l}
x \\
s
\end{array} \mid \lambda\right) K(s, y) d s=D^{*}(\lambda) K(x, y)
\end{array}\right.
$$

zu folgern.

Dieses gelingt in leicht ersichtlicher Weise duroh Anwendung der Schwarzschen Ungleichung bei Berücksichtigung von (27) und (28).

Nennen wir dann $\varphi(x)$ und $\psi(x)$ Lösungen von

$$
\left\{\begin{array}{l}
\varphi(x)-\lambda \int_{a}^{b} K(x, y) \varphi(y) d y=f(x), \\
\psi(x)-\lambda \int_{a}^{b} K(y, x) \psi(y) d y=g(x)
\end{array}\right.
$$

wenn diese Gleichungen für fast alle $x$ erfüllt sind, und betrachten wir ferner zwei Funktionen, die nur in einer Nullmenge voneinander verschieden sind, als nicht verschieden, so können wir aus den Gleichungen (32) und aus den entsprechenden höheren Determinanten (die in derselben Weise zu begründen sind) genau so, wie es in der Arbeit Fredholms geschehen ist ${ }^{y}$ ), die Fundamentalsätze betreffend die Lösbarkeit der Glei-

$\left.{ }^{9}\right)$ Um $z u$ beweisen, daß für eine Wurzel von $D^{*},(\lambda)=0$ nicht alle Determinanten verschwinden können, kann man die Formel (42) benutzen. 
chungen (33) und ihrer zugehörigen homogenen Gleichungen für den Fall beweisen, daß $|f(x)|^{2},|g(x)|^{2}$ und $|K(x, y)|^{2}$ in den Gebieten $a \leqq x \leqq b \quad b z w . \quad a \leqq{ }_{y}^{x} \leqq b$ summierbare Fumktionen sind.

\section{$\$: 3$.}

Um das Geschlecht der ganzen Funktion $D^{*}(\lambda)$ zu bestimmen, wird im nächsten Paragraphen eine Formel für den zu einex Summe von zwei Kernen $G(s, t) \cdot f H(s, t)$ gehörigen Fredholmschen Nenner benutzt, die in meiner Dissertation (Uber das Neumann-Poincarésche Problem. Upsala 1916, S. 9:3) ohne Beweis angegeben wurde.

Diese Formel

$$
\begin{aligned}
& D_{G+\pi}(\lambda)=D_{G}(\lambda) \cdot D_{H}(\lambda)-\lambda^{2} \int_{a}^{b} \int_{a}^{b} D_{G}\left(\begin{array}{l}
s \\
t
\end{array} \mid \lambda\right) D_{H}\left(\begin{array}{l}
t \\
s
\end{array} \mid \lambda\right) d s d t \\
& +\frac{\lambda^{4}}{(\underline{L})^{2}} \int_{a}^{b} \int_{a}^{b} \int_{a}^{b} \int_{a}^{b} D_{a}\left(\begin{array}{l}
s_{1}, s_{2} \\
t_{1}, t_{2}
\end{array} \mid \lambda\right) D_{H}\left(\begin{array}{l}
t_{2}, t_{2} \\
s_{1}, s_{2}
\end{array} \mid \lambda\right) d s_{1} d s_{2} d t_{\mathrm{t}} d t_{\mathrm{z}} \\
& \left.+(-1)^{n} \lambda^{2 n} \int_{(n)^{2}}^{b} \int_{a}^{b} \ldots \int_{a}^{b} D_{a}\left(\begin{array}{c}
s_{1} s_{2} \ldots s_{n} \\
t_{1} t_{2} \ldots t_{n}
\end{array}\right) \lambda\right) D_{H}\left(\begin{array}{c}
t_{1} t_{2} \ldots t_{n} \\
s_{1} s_{2} \ldots s_{n}
\end{array} \mid \lambda\right) d s_{1} \ldots d s_{n} d t_{1} \ldots d t_{n}
\end{aligned}
$$

kann man folgendermaßen beweisen. Zunächst führen wir folgende Bezeichnungen ein. Die zu einem Kern $G$ gehörige Resolvente

$$
\frac{D\left(\begin{array}{l}
x \\
y
\end{array}\right)}{D(\lambda)}
$$

nennen wir: $I_{G}^{\prime}$. Das Resultat der Komposition zweier Kerne $G$ und $H$

wird mit $G H$ bezeichnet.

$$
\int_{a}^{b} G(x, s) H(s, y) d s
$$

Nach einer Formel von Platriér ${ }^{* 0}$ ) ist

$$
\begin{aligned}
& D\left(\begin{array}{l}
x_{1} \\
y_{1}
\end{array} \mid \lambda\right) \ldots D\left(\begin{array}{l}
x_{1} \\
y_{n}
\end{array} \mid \lambda\right)
\end{aligned}
$$

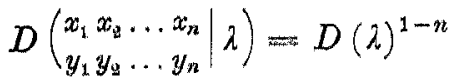

$$
\begin{aligned}
& D\left(\begin{array}{l}
x_{n} \\
y_{1}
\end{array} \mid \lambda\right) \ldots D\left(\begin{array}{l}
x_{n} \\
y_{n}
\end{array} \mid \lambda\right) \\
& D=D(\lambda)\left|\begin{array}{c}
\Gamma\left(x_{1}, y_{1} \mid \lambda\right) \ldots I\left(x_{1}, y_{n} \mid \lambda\right) \\
\Gamma\left(x_{n}, y_{1} \mid \lambda\right) \ldots I\left(x_{n}, y_{n} \mid \lambda\right)
\end{array}\right|
\end{aligned}
$$

10) Journ. de math. 1918, S. 233-304, insb. S. 249. 
Wenden wir ferner die für beliebige Funktionen $\psi_{i}$ und $\psi_{k}$ gültige Formel ${ }^{11}$ )

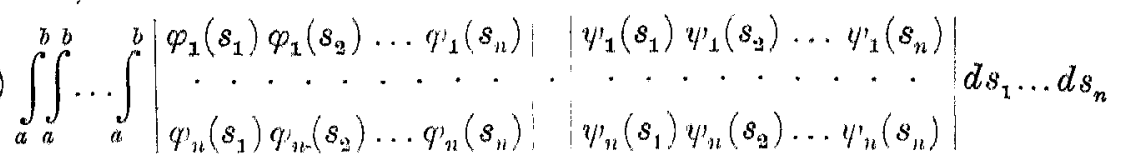

$$
=n \mid \begin{aligned}
& \int_{a}^{b} \psi_{1} \psi_{1} d s, \int_{a}^{b} \varphi_{1} \psi_{2} d s, \ldots, \int_{a}^{b} \psi_{1} \psi_{n} d s \\
& \int_{a}^{b} \psi_{n i} \psi_{1} d s, \int_{a}^{b} \psi_{n} \psi_{2} d s, \ldots . . \int_{a}^{b} \varphi_{n} \psi_{n} d s
\end{aligned}
$$

an, so ergibt sich

$$
\begin{aligned}
& \int_{a}^{b} \int_{a}^{b} \ldots \int_{a}^{b} D_{Q}\left(\begin{array}{c}
s_{1} s_{3} \ldots s_{n} \\
t_{1} t_{2} \ldots t_{n}
\end{array} \mid \lambda\right) D_{H I}\left(\begin{array}{c}
t_{1} t_{2} \ldots t_{n} \\
s_{1} s_{2} \ldots s_{n}
\end{array}\right) d s_{1} d s_{2} \ldots d s_{n} d t_{1} \ldots d t_{n}
\end{aligned}
$$

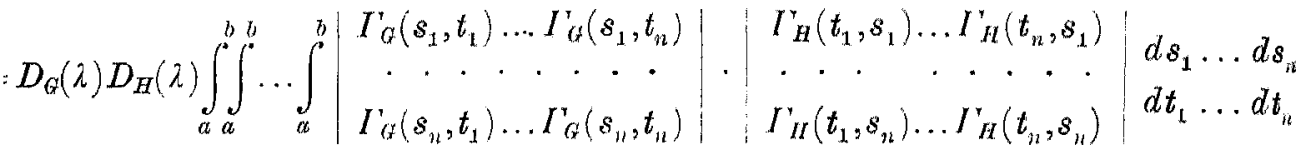

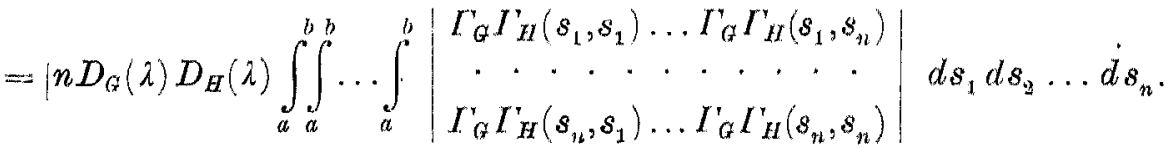

Durch Anwendung dieser Formel finden wir für die rechte Seite von (34) den Ausdruck

$$
D_{G}(\lambda) D_{H}(\lambda) D_{\lambda \Gamma^{\prime}{ }^{\prime}{ }_{H}}(\lambda) \text {. }
$$

Das Problem ist also darauf reduziert, die Relation

$$
D_{G+H}(\lambda)=D_{G}(\lambda) D_{F}(\lambda) D_{\lambda I_{G}^{\prime} I_{H}^{r}}(\lambda)
$$

zu beweisen.

Schreibt man

$$
K(x, y)=K^{\prime}(x, y)+K^{\prime \prime}(x, y)-\lambda \int_{a}^{b} K^{\prime}(x, s) K^{\prime \prime}(s, y) d s,
$$

so gilt nach einem Resultate von Fredholm

Setzen wir hier

$$
D_{K}(\lambda)=D_{R^{\prime}}(\lambda) D_{R^{\prime \prime}}(\lambda)
$$

$$
K^{\prime}=G, \quad K^{\prime \prime}=\lambda \Gamma_{G} \Gamma_{H}
$$

und berücksichtigen, daß

$$
Y_{Q}-G-\lambda Q \Gamma_{G}=0
$$

i) Vgl. G. Landsberg. Math. Ann., 69, S. 281. 
so wird

$$
\begin{aligned}
& K^{\prime}+K^{\prime \prime}-\lambda K^{\prime} K^{\prime \prime}=G+\lambda I_{G} \Gamma_{H}-\lambda^{2} G I_{G}^{\prime} I_{H}=G+\lambda\left(I_{G}-\lambda G I_{G}\right) \Gamma_{H} \\
& \quad \therefore G+\lambda G I_{H}^{\prime},
\end{aligned}
$$

somit

$$
D_{G(}(\lambda) D_{\lambda I_{G I}^{\prime} I_{I I}^{\prime}}(\lambda)=D_{G+\lambda G I_{I I}^{\prime}}
$$

Wenden wir die Formel (38) auf die beiden Kerne $G+\lambda G \Gamma_{H}$ and $H$ an, so ergibt sich wegen

$$
\begin{gathered}
G+\lambda G I_{H}+H-\lambda\left(G+\lambda G \Gamma_{H}\right) H=G+H+\lambda G\left(\Gamma_{H}-H-\lambda \Gamma_{H} H\right) \\
=G+H, . \\
(40) \quad D_{H}(\lambda) D_{G+\lambda G I_{H}^{\prime}}(\lambda)=D_{G+H}(\lambda),
\end{gathered}
$$

woraus schließlich bei Berücksichtigung von (39) die zu beweisende Relation (37) folgt.

Wir erwähnen noch die folgende Formel

$$
\begin{gathered}
D_{(n H}(\lambda) \cdots-J_{(n)^{2}}^{(-\lambda)^{n}} \iint \ldots \int G\left(\begin{array}{c}
s_{1} s_{2} \ldots s_{n} \\
t_{1} t_{1} \ldots t_{n}
\end{array}\right) H\left(\begin{array}{c}
t_{1} t_{2} \ldots t_{n} \\
s_{1} s_{2} \ldots s_{n}
\end{array}\right) d t_{1} \ldots d t_{1} \ldots d t_{n}, \\
G H \cdots \int G(x, s) H(s, y) d s .
\end{gathered}
$$

Hieraus folgt, daß für beschränkte Funktionen $G$ und $H D_{G H}(\lambda)$ höchstens die Ordnung Eins besitzt. Noch allgemeiner gilt

$$
\begin{gathered}
D_{\left(t _ { 1 } \left(t_{2} \ldots a_{m}\right.\right.}(\lambda)=\sum \frac{(-\lambda)^{n}}{(\mid n)^{m}} \iint \ldots \int G_{1}\left(\begin{array}{c}
s_{1}^{(1)} \ldots s_{n}^{(1)} \\
s_{1}^{(2)} \ldots s_{n}^{(2)}
\end{array}\right) G_{2}\left(\begin{array}{c}
s_{1}^{(2)} \ldots s_{n}^{(2)} \\
s_{1}^{(3)} \ldots s_{n}^{(3)}
\end{array}\right) \\
\ldots G_{m}\left(\begin{array}{c}
s_{1}^{(m)} s_{2}^{(m)} \ldots s_{n}^{(m)} \\
s_{1}^{(2)} s_{1}^{(2)} \ldots s_{n}^{(1)}
\end{array}\right) d s_{1}^{(1)} d s_{2}^{(1)} \ldots d s_{n}^{(1)} d s_{1}^{(2)} \ldots d s_{n}^{(2)} \ldots d s_{1}^{(m)} \ldots d s_{n}^{(m)}, \\
G_{1} G_{2} \ldots G_{m} \ldots \iint \ldots \int G_{1}\left(x, s_{1}\right) G_{2}\left(s_{1}, s_{2}\right) \ldots G_{m}\left(s_{m-1}, y\right) d s_{1} d s_{2} \ldots d s_{m-1},
\end{gathered}
$$

woraus folgt, da. $D_{\theta_{1} G_{2} \ldots G_{m}}(\lambda)$ höchstens die Ordnung ${ }_{2}^{m}$ hat, wenn $G_{1} Q_{2} \ldots Q_{m}$ beschränkte Funktionen sind.

Man sieht leicht, wenn man in (34) $G$ und $H$ mit Kernen $G_{1}$ und $H_{1}$ ersetzt, die sich nur dadurch von $Q$ und $H$ unterscheiden, da $B$ sie auf der Diagonale $y \rightarrow x$ verschwinden, daß auch die folgende Formel richtig ist:

$$
\begin{aligned}
& D_{(*+\pi}^{*}(\lambda) \cdots D_{G}^{*}(\lambda) D_{H}^{*}(\lambda)-\lambda^{2} \int_{a}^{b} \int_{a}^{b} D_{G}^{*}\left(\begin{array}{l}
s \\
t
\end{array} \mid \lambda\right) D_{H}^{*}\left(\begin{array}{l}
t \\
s
\end{array} \mid \lambda\right) d s d t \\
& \cdots(-1)^{n} \lambda_{(\underline{n})^{2}}^{\lambda_{a}^{2 n}} \int_{a}^{b} \int_{a}^{b} \ldots \int_{a}^{b} D_{\theta}^{*}\left(\begin{array}{c}
s_{1} s_{2} \ldots s_{n} \\
t_{1} t_{2} \ldots t_{n}
\end{array} \mid \lambda\right) D_{H}^{*}\left(\begin{array}{c}
t_{1} t_{2} \ldots t_{n} \\
s_{1} s_{2} \ldots s_{n}
\end{array} \mid \lambda\right) \begin{array}{l}
d s_{1} \ldots d s_{n} \\
d t_{1} \ldots d t_{n}
\end{array} \ldots
\end{aligned}
$$




\section{$\S 4$.}

Wir wollen nun das Geschlecht der ganzen Funktion $D^{*}(\lambda)$ unter der Annahme bestimmen, daß das Quadrat von $K(x, y)$ im Gebiete $a \leqq y \leqq b$ summierbar ist. $\mathrm{Zu}$ dem Zwecke schreiben wir.

$$
K(x, y)=G(x, y)+H(x, y)
$$

wo $G(x, y)$ von der Form

$$
\sum_{\nu=1}^{m} A_{\nu}(x) B_{\nu}(y)
$$

ist und

$$
\int_{a}^{b} \int_{a}^{b}|H(x, y)|^{2} d x d y<\varepsilon
$$

( $\varepsilon=$ eine beliebig vorgegebene positive Zahl). Dieses kann man z. B. dadurch erreichen, daß man $G(x, y)$ einer geeigneten der früher eingeführten Funktionen $G_{n}(x, y)$ gleichsetzt, wenn das Orthogonalsystem

$$
\Phi_{1}(x, y), \quad \Phi_{2}(x, y) \ldots \Phi_{n}(x, y) \ldots
$$

von der speziellen Form

$$
\varphi_{p}(x) \phi_{q}(y) \quad(p \quad \cdot 1,2, \ldots, q \quad 1,2, \ldots)
$$

gewählt ist, wo $q_{1}(x), \varphi_{2}(x), \ldots, \varphi_{n}(x) \ldots$ ein vollständiges normiertes Orthogonalsystem in bezug auf das Intervall $a \leqq x \leqq b$ bedeutet. Wir wenden nun die Formel (42) an und bemerken, dab diese im vorliegenden Falle nur eine endliche Anzahl Glieder enthält, weil

$$
D_{G}^{*}\left(\begin{array}{c}
s_{1} s_{2} \ldots s_{n} \\
t_{1} t_{2} \ldots t_{n}
\end{array} \mid \lambda\right)=0
$$

für $n>m$. Um eine für unsere $Z$ wecke geeignete obere Grenze von

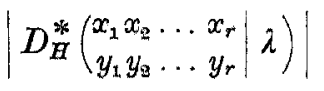

zu erhalten, schreiben wir, zunächst unter der Annahme, daß $H(x, y)$ stetig ist, indem wir uns der Bezeichnungsweise auf S. 7 u. 8 bedienen,

$$
D_{H}^{*}\left(\begin{array}{l}
x_{1} x_{2} \ldots x_{r} \\
y_{1} y_{2} \ldots y_{r}
\end{array}: \lambda\right) \lim _{n \rightarrow \infty} j_{n}
$$




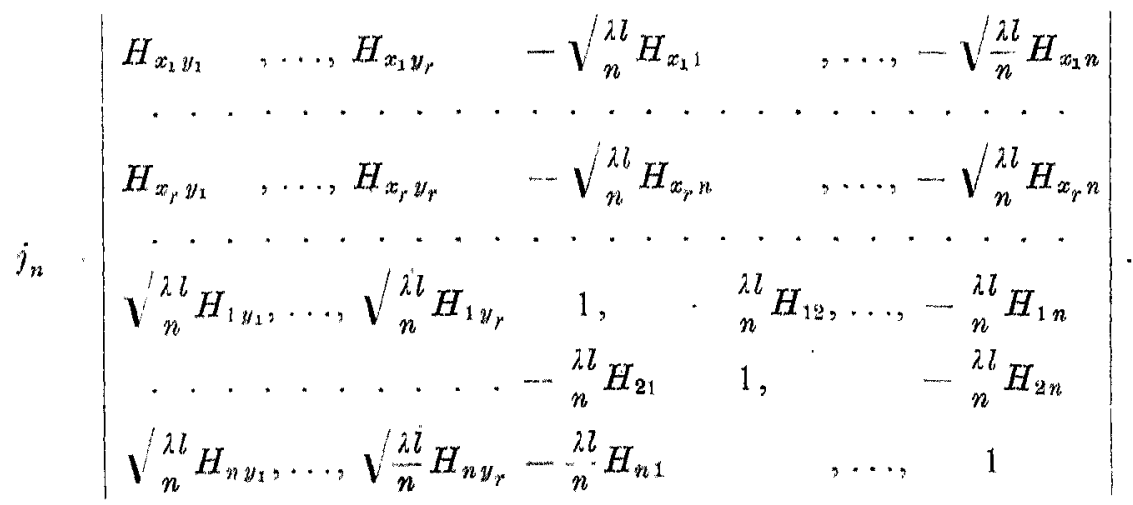

Wir bezeichnen mit $a_{1}, a_{2}, \ldots, \alpha_{e}(\varrho \leqq r) \varrho$ aus den Größen $1,2, \ldots, r$ irgendwie herausgegriffenen verschiedenen Zahlen. Ferner mögen $\gamma_{1}, \gamma_{2}, \ldots, \gamma_{r-e}$ $\left(\gamma_{1}<\gamma_{2}<\ldots<\gamma_{r-0}\right)$ die übriggebliebenen von diesen Größen bezeichnen. Ebenso möge auch $\beta_{1}, \beta_{2}, \ldots, \beta_{Q} ; \delta_{1}, \ldots, \delta_{r-Q}$ ein derartiges System von Zahlen bedeuten. Dann gilt

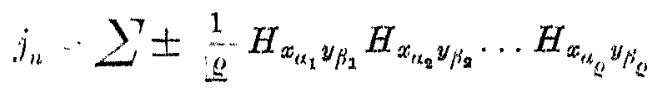

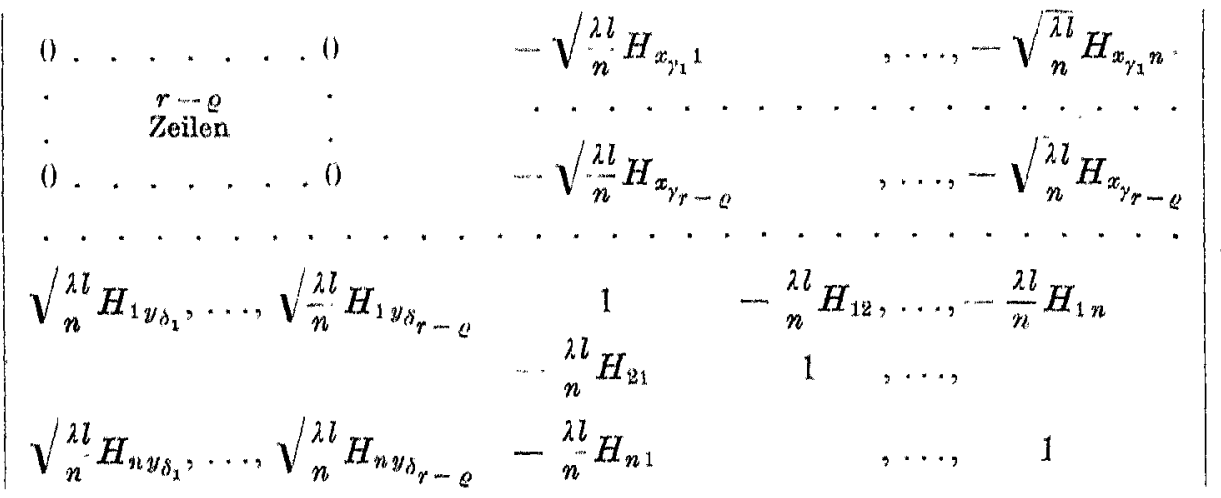

Die Summe ist über alle möglichen Zahlensysteme $a_{1}, \iota_{3}, \ldots, \alpha_{g}$; $\beta_{1}, \beta_{2}, \ldots, \beta_{Q}(\varrho \leqq r)$ zu erstrecken. Hieraus folgt duroh Anwendung des Satzes (18) mit der Bezeichnung

$$
\begin{aligned}
& \sum_{n, 1}^{n}\left|H_{x, 1}\right|^{2} \frac{l}{n}=u_{n}(x)^{2}, \quad \sum_{v=1}^{n}\left|H_{y_{y}}\right|_{n}^{2} l=v_{n}(y)^{2}, \\
& \left|j_{n}\right|<\sum^{\gamma}{ }_{\ell}^{1}\left|H\left(x_{a_{1}}, y_{\beta_{1}}\right) H\left(x_{\pi_{2}}, y_{\beta_{2}}\right) \ldots H\left(x_{a_{\varrho}}, y_{\beta_{Q}}\right)\right| \\
& \cdot|\lambda|^{r-\varrho} u_{n}\left(x_{\gamma_{1}}\right) \ldots u_{n}\left(x_{\gamma_{r-\varrho}}\right) \cdot v_{n}\left(y_{\delta_{1}}\right) \ldots v_{n}\left(y_{\delta_{r-\varrho}}\right) \\
& \left(n+|\lambda|^{2} \sum_{n-r+Q}^{\gamma^{\prime}}\left|H_{p q}\right|^{2} \frac{l^{2}}{n^{2}}\right)^{\frac{n-r+e}{2}}
\end{aligned}
$$


Für $n \rightarrow \infty$ ergibt sich, falls wir schreiben

$$
\begin{aligned}
& \int_{a}^{b}|H(x, t)|^{2} d t=u(x)^{2} ; \quad \int_{a}^{b}|H(t, y)|^{2} d t=v(y)^{2}, \\
& \left|D_{R}^{*}\left(\begin{array}{l}
x_{1} x_{3} \ldots x_{r} \\
y_{1} y_{2} \ldots y_{r}
\end{array} \mid \lambda\right)\right|<\sum \frac{1}{\ell}\left|H\left(x_{a_{1}}, y_{\beta_{1}}\right) \ldots H\left(x_{u_{\ell^{2}}}, y_{\beta_{Q}}\right)\right| . \\
& \cdot|\lambda \sqrt{e}|^{r-e} u\left(x_{\gamma_{2}}\right) \ldots u\left(x_{\gamma_{r-Q}}\right) \cdot v\left(y_{\delta_{2}}\right) \ldots v\left(y_{\gamma_{r-Q}}\right) e \\
& |\lambda|^{2} \int_{a}^{b} \int_{a}^{b}|\boldsymbol{H}(8, t)|^{2} d s d t
\end{aligned}
$$

Durch dieselbe Art von Grenzübergängen, die in dieser Arbeit oft angewandt wurden, zeigt man, daß mit Ausnahme von einer Nullmenge im $2 r$ dimensionalen Raum $a \leqq x_{p} y \leqq b(p \ldots 1,2, \ldots, r, q \cdots 1,2, \ldots, r)$; diese Ungleichung auch in dem Falle gültig bleibt, wo $\mid H(x, y){ }^{2}$ für $a \leqq{ }_{y}^{x} \leqq b$ summierbar ist. Weil es eine Konstante $C$ gibt, daß für $|\lambda|>1$

$$
\left|D_{G}^{*}\left(\begin{array}{l}
x_{1}, x_{2}, \ldots, x_{r} \\
y_{1}, y_{2}, \ldots, y_{r}
\end{array} \mid \lambda\right)\right|<C|\lambda|^{m}
$$

finden wir schließlich aus (42), (4.9) and (45), daß zwei solche (von $\varepsilon$ abhängige) Konstanten $k$ und $N$ existieren, daß für $|\lambda|>1$

$$
\left|D^{*}(\lambda)\right|<k|\lambda|^{N} e^{\frac{v^{*}|\lambda|^{2}}{2}} .
$$

Es seien $\lambda_{1}^{(n)}, \lambda_{2}^{(n)}, \ldots$ die Nullstellen von $D_{G_{n}}^{*}(\lambda)\left(G_{n}(x, y)\right.$ hat hier dieselbe Bedeutung wie auf $\mathrm{S}$. 9). Nach dem schon zitierten Satze von I. $S \operatorname{chur}$ ist

$$
\sum_{v} \frac{1}{\left|\lambda_{y}^{(n)}\right|^{2}} \leqq \int_{a}^{b} \int_{a}^{b}\left|G_{n}(x, y)\right|^{2} d x d y
$$

Durch einen Grenzübergang ergibt sich hieraus für die Nullstellen $\lambda_{1}$, $\lambda_{2}, \ldots, \lambda_{n}, \ldots$ von $D^{*}(\lambda)$

$$
\sum_{1}^{7} \frac{1}{\left|\lambda_{y}\right|^{2}} \leqq \int_{a}^{b} \int_{a}^{b}|K(x, y)|^{2} d x d y
$$

Berüoksichtigen wir noch, dab $D^{*}(\lambda)$ infolge (11) höchstens vom Geschlecht 2 ist, so folgt

$$
D^{*}(\lambda)=e^{a \lambda^{2}+b \lambda} \prod_{\nu=1}^{\infty}\left(1-\frac{\lambda}{\lambda_{\nu}}\right) e^{\lambda_{\nu}}
$$

Auf ganz dieselbe Weise, wie ich in einer früheren Arbeit (Arkiv för mat. astr. o fysik, $\mathrm{Bd} .12,1917)$ eine entsprechende Frage bezüglich $D_{K}(\lambda)$ 
( $K=$ stetiger Kern) behandelt habe, kann man mit Hilfe von (50) schließen, daß

Weil ferner

$$
a=0 \text {. }
$$

$$
D^{*}(0)=1, \quad\left(\frac{d D^{*}(\lambda)}{d \lambda}\right)_{\lambda=0}=0
$$

so ergibt sich aus

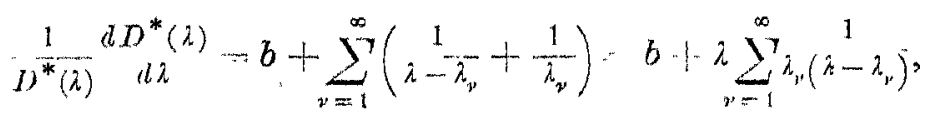

daß $b$ gleich Null ist. Wir haben somit das Resultat gewonnen:

$D^{*}(\lambda)$ ist höchstens vom Geschlechte Eins und besitat die Produktdarstellung

$$
D^{*}(\lambda)=\prod_{r=1}^{\infty}\left(1-\lambda_{y^{\prime}}^{\lambda}\right) e^{\frac{\lambda}{\lambda_{r}}}
$$

(Eingegangen am 19. Januar 1920.) 